\title{
Evolutionary relationships and divergence times among the native rats of Australia
}

\author{
Judith H Robins ${ }^{1 *}$, Patricia A McLenachan², Matthew J Phillips ${ }^{3}$, Bennet J McComish ${ }^{4}$, Elizabeth Matisoo-Smith ${ }^{5}$, \\ Howard A Ross ${ }^{6}$
}

\begin{abstract}
Background: The genus Rattus is highly speciose and has a complex taxonomy that is not fully resolved. As shown previously there are two major groups within the genus, an Asian and an Australo-Papuan group. This study focuses on the Australo-Papuan group and particularly on the Australian rats. There are uncertainties regarding the number of species within the group and the relationships among them. We analysed 16 mitochondrial genomes, including seven novel genomes from six species, to help elucidate the evolutionary history of the Australian rats. We also demonstrate, from a larger dataset, the usefulness of short regions of the mitochondrial genome in identifying these rats at the species level.

Results: Analyses of 16 mitochondrial genomes representing species sampled from Australo-Papuan and Asian clades of Rattus indicate divergence of these two groups $\sim 2.7$ million years ago (Mya). Subsequent diversification of at least 4 lineages within the Australo-Papuan clade was rapid and occurred over the period from 0.9-1.7 Mya, a finding that explains the difficulty in resolving some relationships within this clade. Phylogenetic analyses of our 126 taxon, but shorter sequence (1952 nucleotides long), Rattus database generally give well supported species clades.
\end{abstract}

Conclusions: Our whole mitochondrial genome analyses are concordant with a taxonomic division that places the native Australian rats into the Rattus fuscipes species group. We suggest the following order of divergence of the Australian species. $R$. fuscipes is the oldest lineage among the Australian rats and is not part of a New Guinean radiation. $R$. lutreolus is also within this Australian clade and shallower than $R$. tunneyi while the $R$. sordidus group is the shallowest lineage in the clade. The divergences within the $R$. sordidus and $R$. leucopus lineages occurring about half a million years ago support the hypotheses of more recent interchanges of rats between Australia and New Guinea. While problematic for inference of deeper divergences, we report that the analysis of shorter mitochondrial sequences is very useful for species identification in rats.

\section{Background}

Rattus is the most speciose genus among mammals with some 66 described species [1]. The genus is thought to have originated in the general region of the Indonesian Islands with subsequent dispersal into continental Asia, New Guinea and Australia [2]. Over the last five million years (My) a combination of tectonic and climatic change meant that this geographic region experienced major fluctuations in sea level and in the extent of

\footnotetext{
* Correspondence: j.robins@auckland.ac.nz

'Department of Anthropology and School of Biological Sciences, The

University of Auckland, Auckland, New Zealand Full list of author information is available at the end of the article
}

emergent land mass [3]. These changes provided opportunities for dispersal and speciation within Rattus.

Previous studies $[4,5]$ based on mitochondrial DNA (mtDNA) identified two major groups within Rattus. These are the Asian group, comprising rats from Southeast Asia and Island Southeast Asia, and the AustraloPapuan group comprising rats from Australia and New Guinea. The Asian group includes the three most widely distributed rat species, Rattus rattus and Rattus norvegicus which were dispersed around the world via European sailing ships [6] and Rattus exulans which was transported throughout the Pacific by prehistoric peoples [7]. The Australo-Papuan group of rats are largely restricted to Australia and New Guinea with the
C Biomed Central 
exception of Rattus praetor that also occurs in the Solomon Islands in addition to New Guinea. In addition subfossil remains of this rat have been found at archaeological sites as far east as Fiji indicating that prehistoric peoples in all likelihood transported it into the Pacific [8]. While the Robins et al. [4] study shows good species resolution among the rats of Australia the deeper relationships were not well resolved. The focus of this investigation is to use mtDNA, including whole mitochondrial genomes, to help evaluate these evolutionary relationships and to date the divergences among the Rattus species of Australia.

Rattus has been included in other recent molecular phylogenetic analyses. Jansa et al. [9] investigated relationships among endemic rodents in the Philippine Islands, using single nuclear (exon 1 of IRBP) and mitochondrial (cytochrome $b$ (cyt $b$ )) markers, but they included only one species from the Australo-Papuan group. Rowe et al. [10] sampled broadly among the genera of murine rodents with the aim of inferring relationships among the "Old Endemics" of the AustraloPapuan, or Sahul, region. Their use of six nuclear (exon 10 of GHR, exon 11 of BRCA1, the single large exon of RAG1, intron 3 and flanking regions of BDR, exon 1 of IRBP and intron 2 and flanking regions of ATP5) and six mitochondrial markers (the genes cytochrome c oxidase subunit I (COI), cytochrome $\mathrm{c}$ oxidase subunit II (COII), ATPase 8 and cyt $b$, plus the two tRNA's between COI and ATPase 8) enabled the resolution of deep divergences but their inclusion of only three Rattus species means that there is little overlap with our study. Most recently Pagès et al. [11] developed a large dataset of Rattus and related species based on one nuclear (exon 1 of IRBP) and two mitochondrial markers (cyt $b$ and COI). That study, however, focussed on the species found in Southeast Asia and did not include any of the species endemic to the Australo-Papuan region.

There has been considerable debate regarding the number of rat species in Australia and New Guinea and the relationships among them. Taylor and Horner [12] revised the systematics of the native Australian rats and recognized five species $R$. fuscipes, $R$. leucopus, $R$. lutreolus, $R$. sordidus and $R$. tunneyi. Based on a multivariate analysis of craniometric data Taylor et al. [13] included $R$. fuscipes and R. leucopus within a clade containing New Guinean native rat species whereas the other Australian native rat species ( $R$. sordidus, $R$. tunneyi and R. lutreolus) formed a separate but deeper clade. A phylogeny based on isozyme electrophoresis of 55 loci of the Australian Rattus species, together with $R$. norvegicus and $R$. rattus as outgroups, [14] showed similarities with the phenogram of Taylor et al. [13] with $R$. lutreolus diverging early, followed by $R$. tunneyi and lastly a more recent divergence of a clade containing $R$. sordidus, Rattus colletti and R. villosissimus. This study was unable to resolve the deeper relationships among $R$. fuscipes, $R$. lutreolus and $R$. leucopus and since it did not include rats from New Guinea it could not address the relationship between $R$. fuscipes and the New Guinean rats.

In the recent taxonomic study by Musser and Carleton [1] the rats of Australia and New Guinea are divided into two groups. The Rattus fuscipes species group comprises four of the five Australian native rats described by Taylor and Horner [12] while the fifth, $R$. leucopus, is placed among the New Guinean species in the Rattus leucopus species group. Musser and Carleton [1] and Baverstock et al. [14] give $R$. colletti and $R$. villosissimus full species status whereas Taylor and Horner had classified them both as sub-species of $R$. sordidus. The Rattus leucopus species group of Musser and Carleton [1] comprises the 14 species; Rattus arfakiensis, Rattus arrogans, Rattus giluwensis, Rattus jobiensis, R. leucopus, Rattus mordax, Rattus niobe, Rattus novaeguineae, Rattus omichlodes, $R$. praetor, Rattus richardsoni, Rattus steini, Rattus vandeuseni and Rattus verecundus. Of the Australian and New Guinean species, only two are known to occur in both regions and the results of the craniometric study of Taylor et al. [13] strongly supported the hypothesis of Tate $[15,16]$ that $R$. sordidus colonised southern and south-eastern New Guinea from Australia, and R. leucopus colonised Cape York in north-eastern Australia from New Guinea. The presence of ephemeral land bridges between Australia and New Guinea, especially in the area of the Torres Strait, is believed to be associated with glaciation cycles during the last 2.6 - 3 million years and would have provided opportunities for such faunal interchange $[17,18]$. Aplin (quoted by Musser and Carleton [1] pg 1462), suggested on the basis of preliminary cyt $b$ data that "members of the $R$. fuscipes and the $R$. leucopus groups each may represent discrete radiations, albeit closely related and with some exchange across the Torres strait." Aplin's [17] schematic phylogeny, although it included some New Guinean Rattus species, was similar to that of Baverstock et al. [14]. Aplin placed R. lutreolus basal in the clade containing $R$. tunneyi, $R$. sordidus, $R$. colletti and $R$. villosissimus and did not resolve the position of $R$. fuscipes. He stated that although both positions were uncertain $R$. fuscipes was probably more closely related to the New Guinean rat cluster and $R$. lutreolus to the Australian rat cluster. It is thus fair to say that there is a need for further clarification of the relationships within this speciose group.

In an earlier study Robins et al. [4] used three mitochondrial regions (D-loop, cyt $b$ and COI) to identify Rattus species. Several well-defined clades were recovered. In many cases these corresponded to named 
species, that is all members of the clade had the same species label assigned at source by either the collector or the museum. In some cases, clades contained multiple species labels, possibly resulting from misidentification, faulty taxonomy or biological processes such as introgression. All 16 clades were assigned a name, as shown in Figure two of Robins et al. [4], effectively making them operational taxonomic units (OTUs) although 10 of these names corresponded well to a species. These OTUs are also used in this paper.

From the sample base of the OTUs in Robins et al. [4] six rats were chosen for whole mitochondrial genome analysis and together with data from GenBank divergence times and relationships among five Rattus species were inferred [5]. That 2008 study, however, included only one rat species from the Australo-Papuan region. To increase our understanding of the relationships among the rats of this region we sequenced seven whole mitochondrial genomes from six Rattus species from the Australo-Papuan clade; $R$. fuscipes, $R$. lutreolus, $R$. sordidus, $R$. tunneyi, $R$. villosissimus and two samples of $R$. leucopus (one originating from Australia and one from New Guinea). We have again chosen rats from the sample base of Robins et al. [4] except for the more recently acquired samples of $R$. lutreolus and $R$. villosissimus. We have sequenced whole mitochondrial genomes from four species unique to Australia and two occurring in both Australia and New Guinea. We used these data together with data from GenBank to estimate divergence times and to examine the evolutionary relationships among the Australian rats. We have compared the phylogenies inferred from whole mitochondrial genomes $(\mathrm{n}=16)$ with those obtained by analysing the hypervariable region of the D-Loop together with two mitochondrial gene regions (cyt $b$ and COI) from the same sample set and also from a much larger sample set $(n=$ 126). Here we show that the use of this subset of the mitochondrial genome, while generally reliable for species identification is inadequate for dating or resolving deeper relationships. Our analyses of 16 whole mitochondrial genomes, on the other hand, have made a substantial contribution to our understanding of the relationships among the Australo-Papuan Rattus species and the timing of their divergences.

\section{Methods}

Whole mitochondrial genomes were obtained from seven rats representing six different species (see sample details in Table 1). The species were $R$. fuscipes, $R$. leucopus, $R$. lutreolus, $R$. sordidus, $R$. tunneyi, and $R$. villosissimus. Two specimens of $R$. leucopus (one from New Guinea and the other from Australia) were processed because this species occurs in both New Guinea and in the Cape York region of Australia. These seven novel genomes were compared with other available whole mitochondrial genomes from wild caught rats. There are a number of mitochondrial genomes available from highly inbred strains of $R$. norvegicus (e.g., 10 of the 12 genomes from Schlick et al. [19]) which we did not include in the analysis. DNA was extracted from an additional two samples of $R$. lutreolus and three samples of $R$. villosissimus (see sample details in Table 2). Three mitochondrial regions were sequenced from these samples (750 bp of COI, 762 bp of cyt $b$ and 585 bp of Dloop) and analysed together with the dataset from Robins et al. [4].

DNA was extracted from liver or muscle tissue using the High Pure PCR Template Preparation Kit (Roche). Mitochondrial DNA was amplified in four overlapping long range pieces from 4 to $6 \mathrm{~kb}$ in length. See Table 3 for amplicon length and primer details. Long range PCR was performed using the DNA polymerase Taq and protocols of the Expand Long Template PCR System (Roche). For short-range PCR the amplification reactions contained: TrisHCl pH $8.310 \mathrm{mM}$; KCl $50 \mathrm{mM}$; forward and reverse primers at $0.5 \mu \mathrm{M}$ each; dNTPs at $0.15 \mathrm{mM}$ each; $0.5 \mathrm{U}$ of Taq polymerase; $1 \mu \mathrm{L}$ of DNA template. We used a standard 3-step amplification protocol: $94^{\circ} \mathrm{C}, 3 \mathrm{~min}, 35$ cycles of: $94^{\circ} \mathrm{C}, 30 \mathrm{~s}, 60^{\circ} \mathrm{C}, 30 \mathrm{~s}$, $72^{\circ} \mathrm{C}, 1 \mathrm{~min}$ followed by 1 cycle of $72^{\circ} \mathrm{C}, 5 \mathrm{~min}$ and a hold at $15^{\circ} \mathrm{C}$. PCR products were visualized and quantified on ethidium bromide stained 1\% agarose gels using a low mass ladder from Invitrogen for comparison and purified using either ExoSAP-IT ${ }^{\mathrm{Tm}}$ from USB Corporation or band cut and column cleaned using the QIAquick Gel Extraction Kit $^{\text {tw }}$ from QIAGEN.

All sequencing was done by The Allan Wilson Centre Genome Service at Massey University, Palmerston North, New Zealand. The long range products from each sample except $R$. fuscipes which was sequenced as part of a separate experiment, described in McComish et al. [20] were pooled and processed for sequencing using the multiplexed sequencing kit from Illumina. A 75-bp single read run was performed on an Illumina Genome Analyser GAII (Illumina, Inc.) according to the manufacturer's instructions. After sequencing, the resultant images were analysed with the proprietary Illumina pipeline (version 1.4). This resulted in approximately $964 \mathrm{Mb}$ of sequence, with $90 \%$ of clusters passing the initial filtering step. Reads for each sample were trimmed at the 3 ' end by 5, 10, 15 and 20 bases. Assemblies were made using Velvet 0.7 [21] with a range of hash lengths from 33 to 63 and a minimum k-mer coverage of $5 \times$. Maximum contig lengths and N50 values were tabulated, and the best assembly for each sample was selected for further analysis. Assembled contigs were aligned to the $R$. praetor mitochondrial genome using Geneious 4.7 [22]. 
Table 1 Whole genomes used in this study

\begin{tabular}{|c|c|c|c|c|}
\hline Species & Origin & Source & Specimen Accession & GenBank Accession \\
\hline Microtus kikuchii (vole) & Taiwan & GenBank & & AF348082 \\
\hline Mus musculus domesticus (mouse) & western Europe & GenBank & & NC_006914 \\
\hline R. exulans & New Zealand & EM & RNZAwa01 & EU273711 \\
\hline R. exulans & Thailand & SAM & ABTC 8480 & EU273710 \\
\hline R. exulans & Papua New Guinea & SAM & ABTC 43078 & EU273709 \\
\hline R. fuscipes* & Western Australia, Australia & SAM & ABTC 8615 & GU570664 \\
\hline R. leucopus* & Central Highland, Papua New Guinea & SAM & ABTC 42808 & GU570660 \\
\hline R. leucopus* & Queensland, Australia & SAM & ABTC 51766 & GU570659 \\
\hline R. lutreolus* & Tasmania, Australia & SAM & ABTC 51762 & GU570661 \\
\hline R. norvegicus & Denmark & GenBank & & AJ428514 \\
\hline R. norvegicus & USA & GenBank & & DQ673916 \\
\hline R. norvegicus & Japan & GenBank & & DQ673917 \\
\hline R. praetor & Papua New Guinea & SAM & ABTC 44065 & EU273708 \\
\hline R. rattus & New Zealand & EM & RNZTitRr01 & EU273707 \\
\hline R. sordidus* & Northern Territory, Australia & SAM & ABTC 41164 & GU570665 \\
\hline R. tanezumi & Japan & SAM & ABTC 8514 & EU273712 \\
\hline R. tunneyi* & Northern Territory, Australia & SAM & ABTC 29636 & GU570662 \\
\hline R. villosissimus* & South Australia, Australia & SAM & ABTC 00549 & GU570663 \\
\hline
\end{tabular}

$A n$ * indicates the genomes sequenced as part of this study. EM = Lisa Matisoo-Smith, SAM = South Australia Museum.

Sanger sequencing was used to sequence both ends of each long range fragment to check consistency and to complete the coverage of the mitochondrial genomes. It was also used to sequence the three mitochondrial regions of the additional samples of $R$. lutreolus and $R$. villosissimus. Sanger sequencing was carried out using the BigDye Terminator version 3 sequencing kit, the GeneAmp PCR System 9700 and a capillary ABI3730 DNA Analyser, all from Applied Biosystems. The final assembly of each mitochondrial genome was carried out in Sequencher ${ }^{\text {nix }}$ (Gene Codes Corporation).

\section{Sequence Alignments}

Three main datasets were assembled. The whole genome (WG) dataset (for the alignment see Additional File 1) contained mitochondrial DNA sequences from the genomes of 13 species and included the vole (Cricetidae) and mouse (Muridae) as outgroups (Table 1). The dataset includes 12 mitochondrial genome proteins, rRNA and tRNA coding sequences, totalling 14,471 nucleotides after manual alignment. The D-loop was excluded because of difficulty in alignment. The sequence for protein coding gene ND6 was also excluded because it is encoded on the mitochondrial L-strand. The sequence alignment was partitioned into the three codon positions, RNA stem regions and RNA loop regions. As in the previous work of Robins et al. [5], the third codon positions were coded as purines or pyrimidines (RYcoded) because it was demonstrated there that these positions had a higher substitution rate than the other partitions and substitution models did not sufficiently account for saturation at these sites at deeper levels in the tree. RY-coding greatly reduced saturation in $3^{\text {rd }}$ codon transitions, removed highly significant composition bias and provided better phylogenetic resolution, even within Rattus [5]. Compositional heterogeneity is a recognised problem in mitochondrial genome analysis and for a more detailed discussion of the issues see [23] and other references therein.

A second alignment was assembled from three genomic regions (3G), comprising 126 sequences, spanning $672 \mathrm{bp}$ of cyt $b, 702 \mathrm{bp}$ of the COI and the

Table 2 Additional sequences for the three genomic regions

\begin{tabular}{|c|c|c|c|c|c|}
\hline \multirow[b]{2}{*}{ Species } & \multirow[b]{2}{*}{ Origin } & \multirow[b]{2}{*}{ Specimen Accession } & \multicolumn{3}{|c|}{ GenBank Accession } \\
\hline & & & COI & Cyt $b$ & D-loop \\
\hline R. lutreolus & Myall Lakes, New South Wales & ABTC 51720 & GU570676 & GU570671 & GU570666 \\
\hline R. Iutreolus & Jervois, South Australia & ABTC 27458 & GU570677 & GU570672 & GU570667 \\
\hline R. villosissimus & Purni Bore, South Australia & ABTC 00548 & GU570678 & GU570673 & GU570668 \\
\hline R. villosissimus & Palparara, south west Queensland & ABTC 23632 & GU570679 & GU570674 & GU570669 \\
\hline R. villosissimus & Sir Edward Pellew Is., Northern Territory & ABTC 41137 & GU570680 & GU570675 & GU570670 \\
\hline
\end{tabular}

All specimens were collected in Australia. Tissue samples were provided from specimens by the South Australia Museum. 
Table 3 The primers used to amplify the four overlapping long range amplicons

\begin{tabular}{|c|c|c|}
\hline \multicolumn{3}{|l|}{ A. } \\
\hline Primer name & \multicolumn{2}{|l|}{ Sequence $\left(5^{\prime}\right.$ to $\left.3^{\prime}\right)$} \\
\hline Av175312SF & \multicolumn{2}{|c|}{ AAACTGGGATTAGATACCCCACTAT } \\
\hline R6036R & \multicolumn{2}{|c|}{ ACTTCTGGGTGTCCAAAGAATCA } \\
\hline BatL5310 & \multicolumn{2}{|c|}{ CCTACTCRGCCATTITACCTATG } \\
\hline LR2RB & \multicolumn{2}{|c|}{ CTGATTGGAAGTCAGTTGTATIIT } \\
\hline L10647F & \multicolumn{2}{|c|}{ TTTGAAGCAGCAGCCTGATAYTG } \\
\hline $\mathrm{RCb9H}$ & \multicolumn{2}{|c|}{ TACACCTAGGAGGTCTITAATTG } \\
\hline RGlu2L & \multicolumn{2}{|c|}{ CAGCATTTAACTGTGACTAATGAC } \\
\hline Long 16SR & \multicolumn{2}{|c|}{ TGATTATGCTACCTITGCACGGTCAGGATACC } \\
\hline \multicolumn{3}{|l|}{ B. } \\
\hline Region amplified & Primer pairs & Amplicon length \\
\hline $12 \mathrm{~S}-\mathrm{COI}$ & Av175312sf \& R6036R & $5.55 \mathrm{~kb}$ \\
\hline COI - COIII & BatL5310 \& LR2RB & $4.11 \mathrm{~kb}$ \\
\hline COIII - Cyt b & L10647F \& RCb9H & $5.53 \mathrm{~kb}$ \\
\hline Cyt $b-16 S$ & RGlu2L \& Long 16SR & $4.26 \mathrm{~kb}$ \\
\hline
\end{tabular}

(A) The primer sequences $\left(5^{\prime}\right.$ to $\left.3^{\prime}\right)$. (B) The primer pairs used for each genome region and the amplicon length.

hypervariable region of the D-loop, for a total length of 1952 bp. This dataset which includes Mus as the outgroup extends that of Robins et al [4] by the inclusion of sequences from seven new samples, three of $R$. lutreolus and four of R. villosissimus (for sample details see Tables 1 and 2). The vole outgroup was not included because of the difficulty in aligning the D-loop. This alignment was analysed in three ways: with three partitions (codon positions $1+2$, codon position 3 as RY, and all other positions see Additional File 2), with each genomic region as a separate but unencoded partition (Additional File 3), and as a concatenated sequence with no partitions. The coding of sequence labels in the nexus files to species names and GenBank accessions is given in Additional File 4.

A third alignment (3G-WG) was created (Additional File 5) by extracting the $3 G$ regions from the specimens in the WG dataset. This enabled us to assess whether the differences in the results obtained using the WG and $3 G$ datasets were due to the specimens or to the genomic regions used.

Of the gene regions that we have sequenced, cytochrome $b$ is the most widely represented among rodent species in GenBank. To test whether the AustraloPapuan clade was monophyletic we assembled a rodent alignment of cytochrome $b$ sequences from 245 representatives of Rattus sensu lato [24] (see Additional File 6). This included our data as well as additional data deposited in GenBank by Pagès et al. [11], Jansa et al. [9], and Rowe et al. [10]. The sequences from the various studies tended to be either $\sim 700$ or $\sim 1200 \mathrm{bp}$ in length. Further we wished to use both unencoded and
RY-encoded data. Consequently we created four alignments. The first used the complete alignment from Additional File 6 (1-1182 bases), and the second used a short alignment (bases 1-713). In the third and fourth alignments the data from both the complete and the short alignment were partitioned into codon positions 1 +2 (unencoded) and codon position 3 (coded as RY). These codon-partitioned alignments were trimmed by 40 bases at the 3' end to remove the non-coding tRNA region from the sequences leaving bases 41-1182 and 41 - 713 respectively.

\section{Phylogenetic methods}

The optimal substitution model was determined in previous work [5] to be GTR + I $+\mathrm{G}_{4}$. This model was used in partitioned maximum likelihood (ML) and Bayesian inference analyses. ML analyses were performed on the WG dataset with PAUP* [25] and RAxML [26]. Other exploratory ML analyses were performed using PHYML [27]. Phylogenetic trees were estimated from the WG, 3G, 3G-WG and the four rodent cytochrome $b$ alignments under Bayesian inference with MrBayes [28]. For the WG dataset, three Markov chain Monte Carlo (MCMC) chains for each of two independent runs proceeded for 8,500,000 generations with trees being sampled every 5000 generations. The burnin length $1,500,000$ was determined by examination of $-\ln L$ versus MCMC generation plots and sampling efficiency within Tracer 1.5 [29]. For the 3G and 3G-WG datasets, three MCMC chains for each of two independent runs were sampled for 10,000,000 generations with trees sampled every 1000 generations. The burnin length of 100,000 generations was chosen by the same method.

In addition to the three phylogenetic trees estimated from each of the WG, 3G and 3G-WG datasets, estimations were made using ML for alignments based on single gene regions from both the WG and 3G datasets. In all, 18 tree topologies were obtained for the species represented in the WG dataset. Support in the WG dataset among these alternative topologies was examined using the Kishino-Hasegawa (KH) [30] and approximately unbiased (AU) [31] tests within the CONSEL program [32]. Sitewise log likelihoods were combined from the GTR $+\mathrm{I}+\mathrm{G}_{4}\left(\mathrm{CF} 87+\mathrm{I}+\mathrm{G}_{4}\right.$ for RY-coded data) maximum likelihood (ML) analyses run in PAUP* for each of the separately run partitions.

We assessed variation along the genome in the strength of support for the two main topologies, that with the clade comprising $R$. lutreolus, $R$. sordidus, $R$. tunneyi and $R$. villosissimus (Figure 1 ) or that with the clade comprising $R$. fuscipes, $R$. leucopus and $R$. praetor (Figure $2 \mathrm{~B}$ ) as a more recent divergence. The whole genome alignment was subdivided into $1000 \mathrm{bp} \mathrm{sec-}$ tions, and an ML bootstrap analysis performed on 


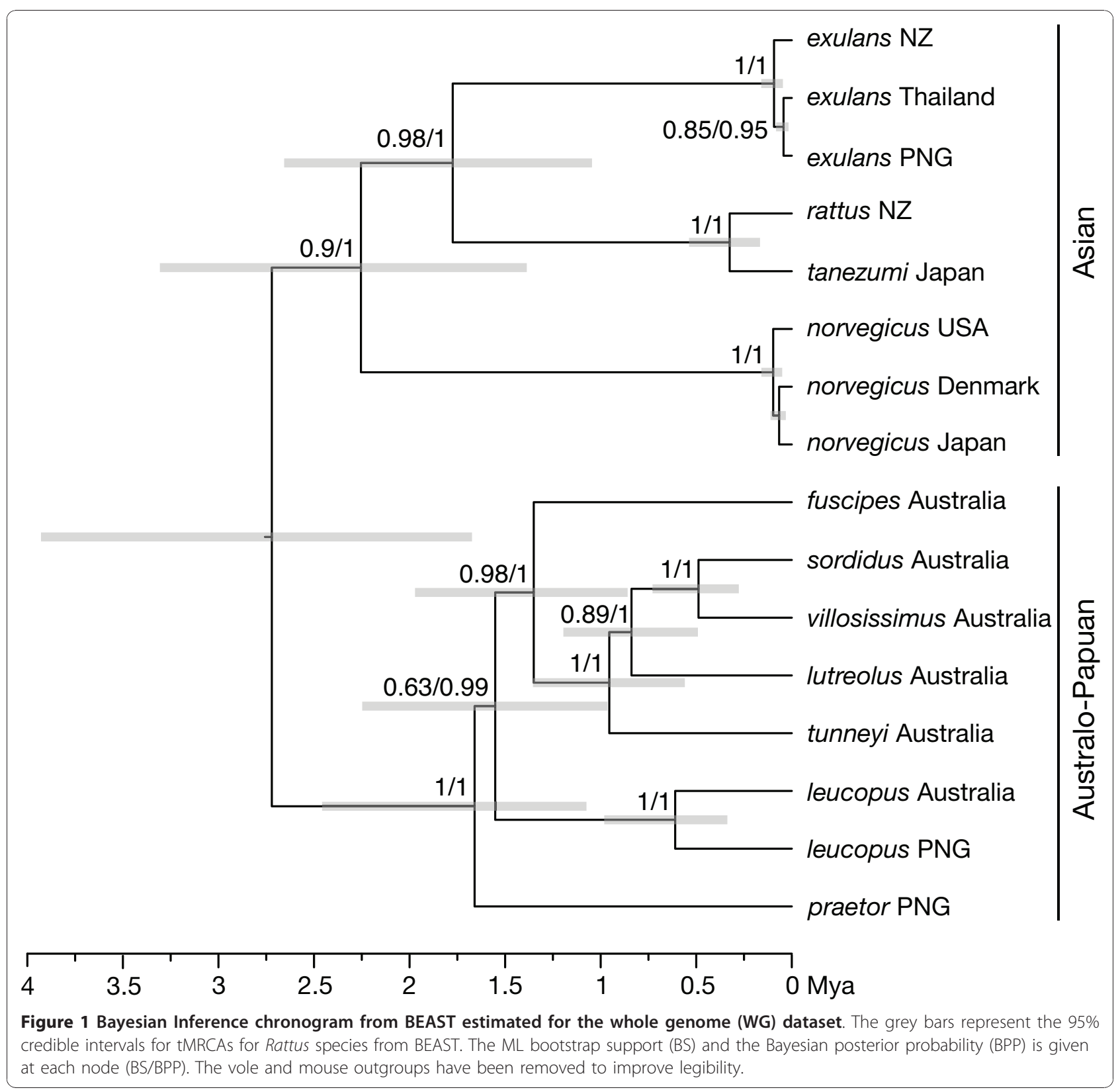

each non-partitioned section using PHYML and the GTR $+\mathrm{G}+\mathrm{I}$ model of evolution. We estimated the frequency of each clade for each region among the bootstrap trees.

BEAST [33] was used to apply Bayesian inference to the estimation of divergence dates within the phylogenies of the WG, 3G and 3G-WG species. For the WG dataset, as in previous work [5], a partitioned analysis was performed in which codon positions 1 and 2 were combined, RNA stem and loop partitions were combined and codon position 3 was coded as RY, to counteract the effects of rate heterogeneity and substitution saturation at hypervariable sites. The MCMC chain was run for 10,000,000 generations, with both trees and ages sampled every 5,000 generations. The "relaxed" clock method was used in which rates along branches are distributed according to a lognormal distribution [34]. For the $3 \mathrm{G}$ and $3 \mathrm{G}-\mathrm{WG}$ datasets, a partitioned analysis was performed in which codon positions 1 and 2 were combined, codon position 3 was coded as RY and all other sites formed the third partition. A birth-death model of speciation was used with the WG and 3G-WG datasets, in which each species is represented by a single sequence, whereas a coalescent model was used with the $3 \mathrm{G}$ dataset, in which each species has multiple specimens. The sampled trees were summarised as maximum 

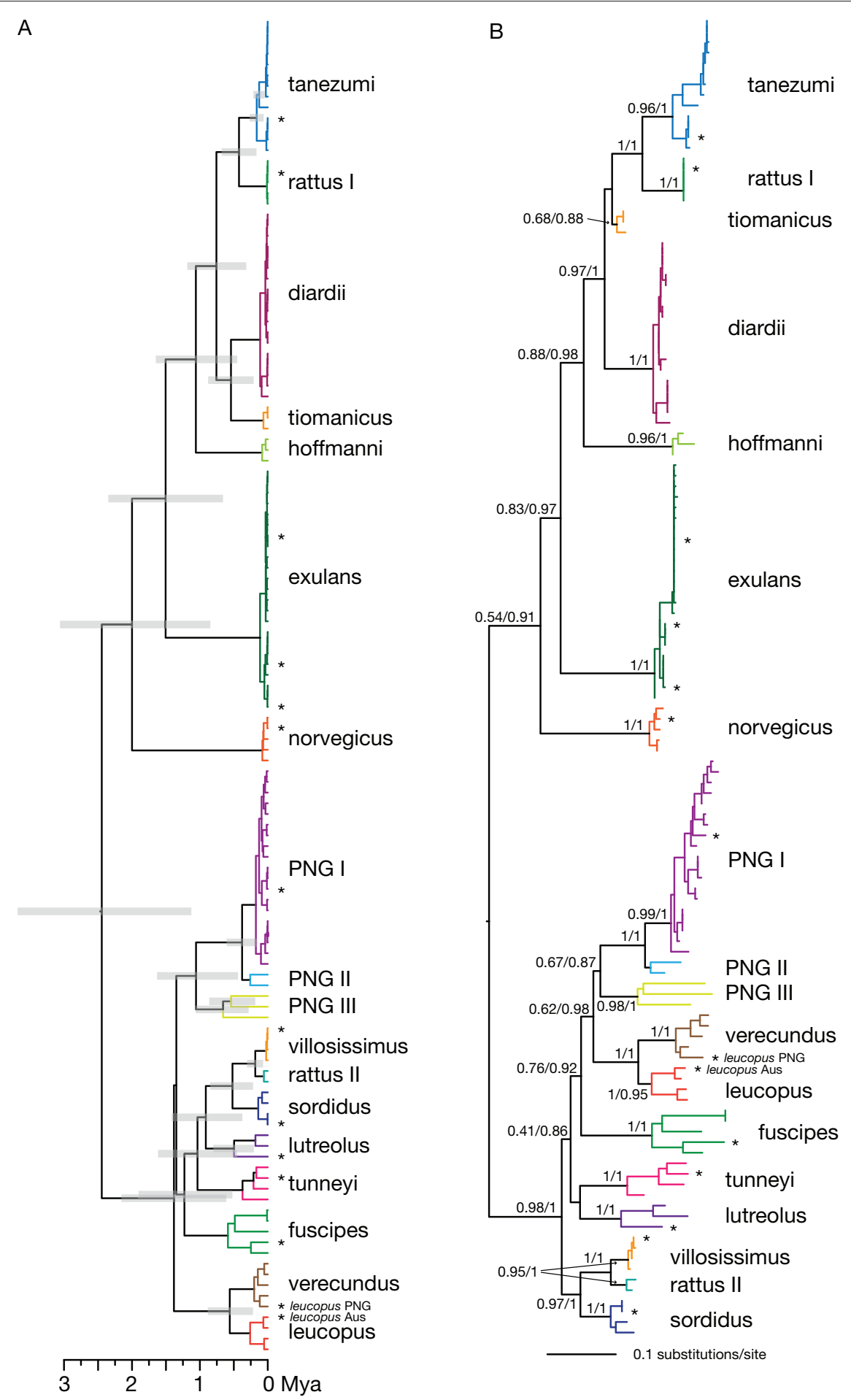

Figure 2 (A) Bayesian Inference chronogram and (B) ML and MrBayes tree estimated for the three genome region (3G) dataset. Clades are individually colored, and labelled according to the "phylogenetic species" identified in Robins et al [4]. The grey bars represent the $95 \%$ credible intervals for tMRCAs for Rattus clades from BEAST. In the BEAST tree (A), the outgroup has been removed, the Asian and the AustraloPapuan clades are constrained to be monophyletic and a prior is assigned to the root age as described in the text. The ML and MrBayes tree (B) is labelled with bootstrap support (BS) and the Bayesian posterior probability (BPP) at each node (BS/BPP). The mouse outgroup has been removed to improve legibility. Sequences marked with * are from the WG dataset. 
clade credibility trees with node heights set to the median of sampled values using TreeAnnotator [33].

We applied age constraints on two nodes to calibrate our BEAST analysis of the WG dataset: the split between Muridae (mouse plus rats) and Cricetidae (vole), that is the root node, and the split between Mus and Rattus within Muridae. As in previous work [5] we used the recommendations of Benton and Donoghue [35] for the minimum and maximum age constraints of the mouse-rat divergence. They state that current research suggests that the split between Mus and Rattus is early in the evolution of Murinae but not basal in the divergence of the clade. They recommend a lower bound of 12.3 Mya since this is the oldest record of Progonomys, the genus assumed to contain the common ancestor of Mus and Rattus and an upper bound of 11 Mya which is based on records of the extinct genus Karnimata which is believed to be on the lineage leading to Rattus. These bounds were used respectively for the middle $95 \%$ of the normally distributed prior. The Mus plus Rattus clade was enforced relative to the vole which is in agreement with all recent molecular and morpological interpretations. A uniform prior from 11 to 34 Mya for Muridae (mouse and rats) versus Cricetidae (vole) provided a calibration on the root that is very conservative at both the upper and lower bounds (see [36]).

The chronogram obtained (as described above) from BEAST for the 3G dataset contained unrealistically old ages for the nodes in the tree and a topology that differed markedly from that estimated for the WG dataset. Consequently, to test the effect of substitutional saturation, a further BEAST analysis was performed using a coalescent prior and constant population size. Here, the Mus outgroup was removed because of the long branch lengths between Mus and Rattus and potential saturation in the hypervariable region of the D-loop. The Asian and Australo-Papuan clades were each constrained to be monophyletic and the age of the root, the age of the most recent common ancestor (tMRCA) for Rattus, was assigned a lognormal prior based on the empirical results from the WG analysis.

\section{Results \\ Topology}

Three datasets were used for phylogenetic reconstructions. These differed in their extent of taxon sampling and in their sequence length (whole mitochondrial genomes (WG): 16 taxa 16293-16309 characters; three genomic regions (3G): 126 taxa 1952 characters; whole mitochondrial genomes trimmed to three regions (3GWG): 16 taxa 1952 characters). As described below, similar but different topologies were inferred for relationships among the Asian clade when the larger taxon dataset was used. Differences in the relationships among Australian species were also inferred when New Guinean sequences were included in the analysis of Australian species. The greatest uncertainty in relationships among the Australian species was due to different root placements being optimal for the Australo-Papuan clade in some analyses of the different datasets. As we discuss, these observations are consistent with expectations for rapid speciation $[37,38]$.

\section{The whole genome (WG) dataset}

The trees estimated by ML and Bayesian inference from the WG dataset have the same topology (Figure 1) with the minor exception of differences in the branching order among the $R$. norvegicus sequences. The topological relationships within the clade of Asian species $(R$. exulans, $R$. norvegicus, $R$. rattus and $R$. tanezumi) are the same as previously recovered [5]. The AustraloPapuan species ( $R$. fuscipes, $R$. leucopus, $R$. lutreolus, $R$. praetor, $R$. sordidus, $R$. tunneyi and $R$. villosissimus) form a sister clade to the Asian clade. Overall eighteen hypotheses regarding the relationships among the Australo-Papuan clade of Rattus were tested using the CONSEL program. The log-likelihood differences between the optimal topology and the other hypotheses of relationship for the WG data are given in Table 4 . The relationships represented in Figure 1 had the highest likelihood, but four other topologies were clearly not significantly poorer and a fifth was only marginally not significantly poorer. Common features of the five best trees include a) the early divergence of $R$. praetor and $R$. leucopus from the other lineages, and $\mathrm{b}$ ) the late divergence of $R$. sordidus and $R$. villosissimus. Trees in which $R$. praetor diverge late (e.g. 12, 16, 18 ) are much poorer than the best trees.

The Australo-Papuan clade has strong support from both ML and Bayesian inference (Figure 1). Within the Australo-Papuan clade, the analyses of the WG dataset indicate support for $R$. sordidus and $R$. villosissimus as sister taxa, with the inclusion of $R$. lutreolus, $R$. tunneyi and then $R$. fuscipes as one moves back in time. The order of divergence of $R$. praetor and R. leucopus is somewhat uncertain; the time interval between the dates of divergence is small and ML bootstrap support is weak.

Three mitochondrial gene (3G) region (Cyt b, COI, D-Loop) datasets

Phylogenetic analysis of the $3 \mathrm{G}$ dataset obtained from ML and MrBayes analyses, when the codon positions are partitioned and RY encoding used, returned the same topology (Figure 2B) corresponding to topology 12 in Table 4 where, as a member of the PNG I clade, $R$. praetor diverges recently and $R$. lutreolus, $R$. sordidus, $R$. tunneyi and $R$. villosissimus have more basal positions. This topology is not supported by the WG dataset (see Figure 1). 
Table 4 Log-likelihood differences between trees

\begin{tabular}{|c|c|c|c|c|}
\hline & \multirow[t]{2}{*}{ Tree topologies } & \multirow[t]{2}{*}{$-\ln L$} & \multicolumn{2}{|c|}{$P$ values } \\
\hline & & & $\overline{A U}$ & KH \\
\hline 1 & (Out, (AR,(((((Rsor,Rvil),Rlut),Rtun),Rfus),Rleu),Rpraet))) & $<41273.6>$ & - & - \\
\hline 2 & (Out, $(\mathbf{A R},((((($ Rsor,,Rvil),Rlut),Rtun),Rfus),Rpraet),Rleu $)))$ & 2.6 & 0.556 & 0.351 \\
\hline 3 & (Out, $(\mathbf{A R},(((($ Rsor,Rvil),Rlut),Rtun),Rfus),(Rleu,Rpraet)))) & 4.1 & 0.393 & 0.254 \\
\hline 4 & (Out,,$(\mathbf{A R},(((($ Rsor,Rvil),(Rlut,Rtun)),Rfus),Rpraet),Rleu $)))$ & 9.3 & 0.208 & 0.170 \\
\hline 5 & (Out, $(\mathbf{A R},((($ Rsor,Rvil),(Rtun,Rlut)),Rfus),(Rleu,Rpraet)))) & 11.2 & 0.144 & 0.126 \\
\hline 6 & (Out, $(\mathbf{A R},(((($ Rsor, Rvil),Rlut),Rtun),(Rleu,Rfus)),,Rpraet))) & 16.3 & 0.082 & 0.054 \\
\hline 7 & (Out, $(\mathbf{A R},((((($ Rsor,,Rvil),Rlut),Rtun),Rleu),Rpraet),Rfus $)))$ & 25.8 & $0.020^{*}$ & $0.018^{*}$ \\
\hline 8 & (Out, $(\mathbf{A R},(((($ Rsor,Rvil),Rtun),Rlut),(Rfus,Rpraet)),Rleu $)))$ & 30.9 & $0.019^{*}$ & $0.018^{*}$ \\
\hline 9 & (Out,,$(\mathbf{A R},(((($ Rsor, Rvil),Rtun),Rlut),((Rleu,Rfus),Rpraet)))) & 31.2 & $0.028^{*}$ & $0.021^{*}$ \\
\hline 10 & (Out, $(\mathbf{A R},((($ Rsor, Rvil),(Rtun,Rlut)),((Rfus,Rpraet),Rleu $)))$ & 34.0 & $0.025^{*}$ & $0.013^{*}$ \\
\hline 11 & (Out,,$(\mathbf{A R},(((($ Rsor, Rvil),Rtun),Rlut),((Rleu,Rfus),Rpraet)))) & 101.9 & $>0.001^{*}$ & $>0.001^{*}$ \\
\hline 12 & (Out, $(\mathbf{A R},(($ Rsor,Rvil),((Rlut,Rtun),(Rfus,(Rleu,Rpraet) $)))))$ & 133.8 & $>0.001^{*}$ & $>0.001^{*}$ \\
\hline 13 & (Out, $(\mathbf{A R},((($ Rsor,Rtun),Rvil),(Rlut,Rfus)),(Rleu,Rpraet)))) & 199.0 & $>0.001^{*}$ & $>0.001^{*}$ \\
\hline 14 & (Out, $(\mathbf{A R},(((($ Rsor, Rvil),Rleu),Rfus),(Rtun,Rlut)),Rpraet))) & 208.6 & $>0.001^{*}$ & $>0.001^{*}$ \\
\hline 15 & (Out, (AR,((((Rsor,Rvil),Rlut),Rtun),(Rfus,(Rleu,Rpraet))))) & 274.2 & $>0.001^{*}$ & $>0.001^{*}$ \\
\hline 16 & (Out,,$(\mathbf{A R},(((($ Rsor, Rvil),,Rlut),Rtun),(Rpraet,Rfus)),Rleu $)))$ & 277.7 & $>0.001^{*}$ & $>0.001^{*}$ \\
\hline 17 & (Out, $(\mathbf{A R},(((($ Rsor,Rlut),Rtun),((Rvil,Rfus),Rpraet)),Rleu $)))$ & 343.5 & $>0.001^{*}$ & $>0.001^{*}$ \\
\hline 18 & (Out,,$(\mathbf{A R},(($ Rsor,Rvil),((Rlut,Rtun),((Rfus,Rpraet),Rleu $)))))$ & 409.9 & $>0.001^{*}$ & $>0.001^{*}$ \\
\hline
\end{tabular}

The relationships among Australo-Papuan Rattus species and their statistical significance under AU and $\mathrm{KH}$ tests. The column headed -InL gives the log-likelihood of the best tree in the first line and the differences in log-likelihood of the other trees in subsequent lines. ${ }^{*}$ indicates $P \leq 0.05$. Taxon abbreviations: Rex $R$. exulans, Rfus $R$. fuscipes, Rleu $R$. leucopus, Rlut $R$. lutreolus, Rnov $R$. norvegicus, Rpraet $R$. praetor, Rrat $R$. rattus, Rsor $R$. sordidus, Rtan $R$. tanezumi, Rtun $R$. tunneyi, Rvil R. villosissimus. Out represents the non-Rattus Mouse and Vole and AR the Asian Rattus clade(((Rex),(Rrat, Rtan)),(Rnov)). Rex comprises the taxa RexNZ, RexThai, RexPNG; Rnov comprises the taxa RnovDen, RnovUSA, RnovJap; Rleu comprises the taxa Rleu Australia, Rleu Papua New Guinea.

A slightly different topology again was found when the $3 G$ dataset was analysed with MrBayes without RY encoding, either with the alignment partitioned by region or simply concatenated. Here, the order of divergence of $R$. leucopus and $R$. praetor is reversed, and $R$. lutreolus and $R$. tunneyi form a clade. This corresponds to topology 4 of Table 4.

The major differences in tree topology inferred from the $3 \mathrm{G}$ dataset, as compared with the WG dataset, may derive from the impact of the many additional sequences in the $3 \mathrm{G}$ dataset or from the reduction in the number of genomic regions and partitions, and the inclusion of the hypervariable D-loop. To test this possibility, the 3G dataset was pruned of all sequences not in the WG dataset. For the tree returned by a BEAST analysis of this 3G-WG dataset (Figure 3A; topology 16 in Table 4), the unrooted topology of the Australo-Papuan clade is highly similar to that shown in Figure 1. It differs only in the position of $R$. praetor, however, the root of the Australo-Papuan clade is placed differently. Both ML and MrBayes estimations of the phylogenetic tree produced a topology (Figure $3 \mathrm{~B}$ ) with notable differences in the positions of the root of the AustraloPapuan clade and of R. tunneyi (topology 18 in Table 4).

The frequency along the genome for the two topologies, that with the clade comprising $R$. lutreolus, $R$. sordidus, $R$. tunneyi and $R$. villosissimus (Figure 1 ) or that with the clade comprising $R$. fuscipes, $R$. leucopus and $R$. praetor (Figure $2 \mathrm{~B}$ ) as a more recent divergence is summarized in Figure 4. The support for these clades is highly variable along the genomes. The fragment containing the D-loop provided the strongest support for the ( $R$. fuscipes, $R$. leucopus and $R$. praetor) clade. Further, it contained strong support for the (R. lutreolus, $R$. sordidus, $R$. tunneyi and $R$. villosissimus) clade. The sections containing the COI and Cyt $b$ genomic regions provided only weak support for either of these clades.

Another approach to testing the effects of the gene regions used was to remove the outgroup and constrain the height of the tree. This will reduce the possibility of misplacing the root of the Australo-Papuan clade as a result of long branch attraction relative to the outgroup and stochastic artefacts. It will also diminish the influence of hypervariable regions of the sequence. The topology returned from this analysis (Figure 2A) was very similar to, and not significantly different from, that obtained in the WG analysis (Figure 1), corresponding to topology 2 , or marginally topology 1 , in Table 4 .

In summary, both the $3 \mathrm{G}$ and $3 \mathrm{G}-\mathrm{WG}$ datasets gave topologies significantly different from that obtained using the WG dataset when Bayesian inference (both BEAST and MrBayes) or maximum likelihood was used to analyse partitioned and RY-coded datasets. Reducing the effects of the outgroup or the hypervariable regions enabled us to 


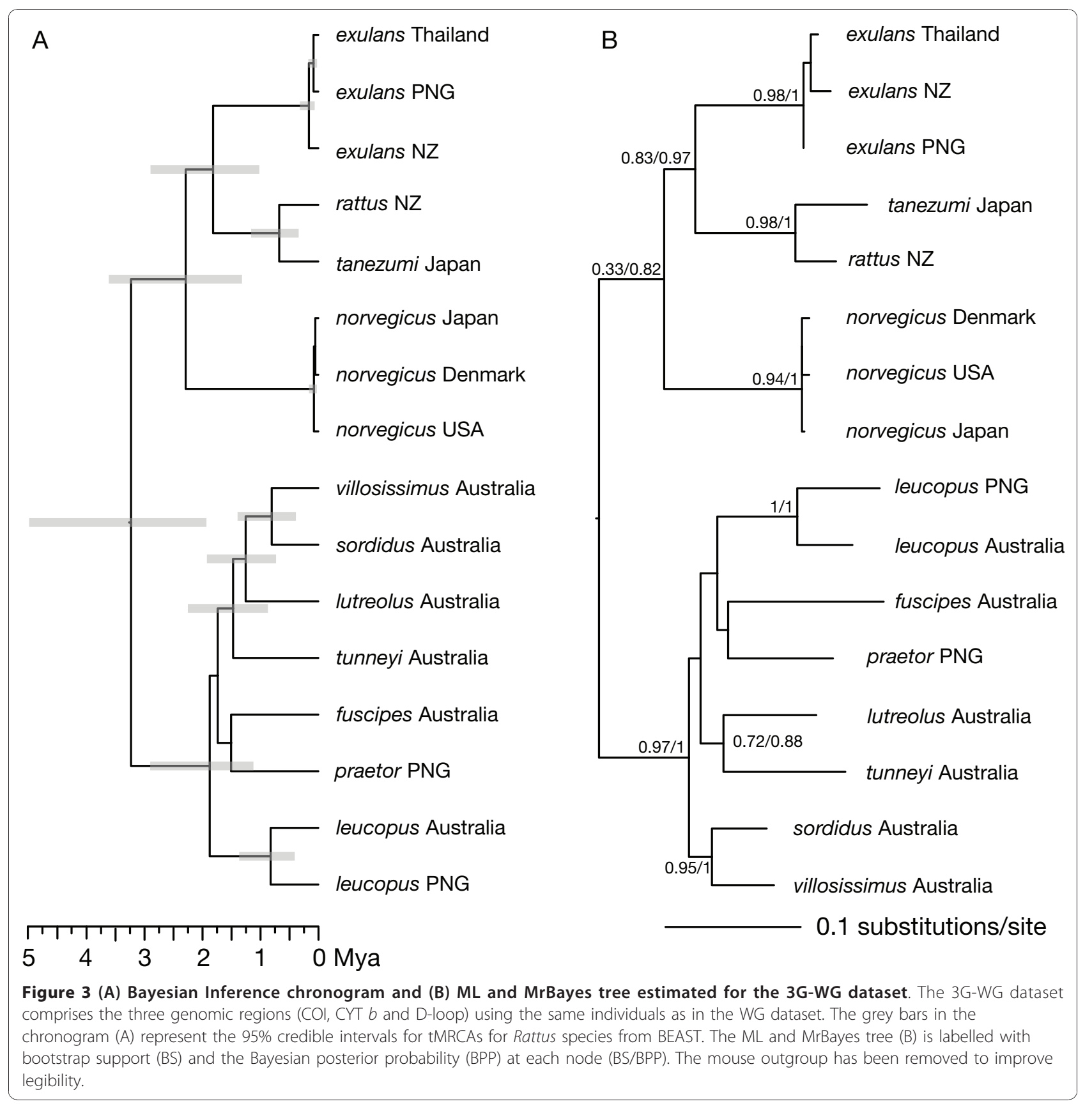

recover the WG topology. Consequently we can infer that it was the use of only these three limited genomic regions, rather than the inclusion of many additional sequences, that led to the inference of a different topology from the $3 G$ dataset than from those of the pruned 3G-WG and the WG datasets. Nevertheless our analyses of the $3 G$ dataset provided generally well supported species clades which are useful for species identification.

The four cytochrome $b$ alignments returned very similar results. We provide the tree with species clades collapsed for greater legibility in Additional File 7 and as a full tree file in Additional File 8. In each analysis the order of divergence was Maxomys deepest followed by Micromys, Niviventer and Leopoldamys. The AustraloPapuan rats always formed a well-supported monophyletic clade. The clades representing Bandicota, Berylmys, Tarsomys, Limnomys, $R$. norvegicus and its sister species $R$. nitidus, and the remaining clade of Asian rats were each observed consistently with high support. However, the relationships among them were unstable and were not resolved with high posterior probability or bootstrap support (results not shown). 


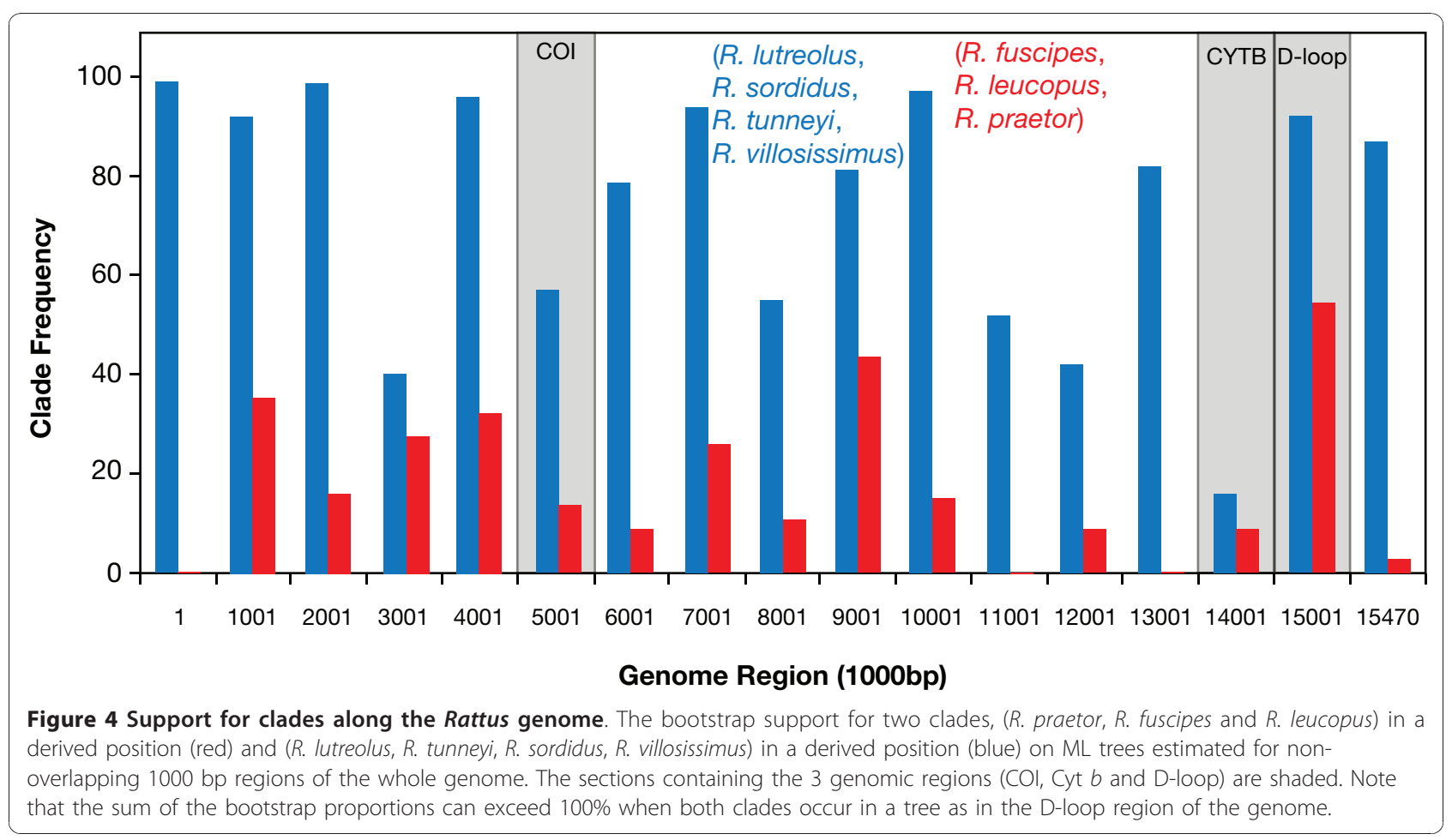

\section{Dates of Divergence}

The addition of seven genomes has made more recent the estimated MRCAs within the Rattus genus when compared with previous work [5]. The median age of the split between the Asian and Australo-Papuan clades is estimated to be 2.7 Mya (95\% credible interval 1.7 3.9 Mya). Our previous estimate of this date, based on eight Asian rat genomes but only a single genome from the Australo-Papuan clade ( $R$. praetor), was near the upper bound at 3.8 Mya. Similarly, the previous estimate of tMRCA of the Asian clade, at 3.1 Mya, falls near the upper bound of the estimate obtained here with median 2.3 and 95\% credible range 1.4 - 3.3 Mya. The MRCA for the Australo-Papuan clade is $1.66 \mathrm{Mya}$, and the most recent divergence within that clade, between $R$. sordidus and $R$. villosissimus, is estimated to be approximately 500,000 ya (Table 5 ).

When the dates of divergence were estimated for the $3 G$ dataset, nodes in the tree were more than twice as old as when estimated using the WG dataset. However, when the MRCA for Rattus was constrained, the ages of the divergences in the Australo-Papuan clade (Figure 2A) more closely resembled those obtained from the WG dataset.

The estimates of the node ages obtained using the 3GWG dataset (Figure 3) are comparable but slightly older than those obtained from the whole genome. The Asian - Australo-Papuan split is at 3.2 Mya as compared with 2.7 Mya, and the Australo-Papuan clade has a MRCA of
1.87 Mya as compared with 1.66 Mya from the whole genome. In contrast, the deeper splits within the Asian clade have nearly the same estimated age. The divergence of $R$. norvegicus is estimated to be $2.28 \mathrm{Mya}$, as compared with 2.25 Mya, and the divergence of $R$. exulans from the other Rattus at 1.81 versus 1.77 Mya when the whole genome was used.

\section{Discussion}

Whole mitochondrial genomes have been used to infer phylogenetic relationships in a wide range of organisms e.g., mammals [39-43], birds [44], fish [45] and worms [46]. Phylogenies based on mitochondrial genomes are effectively gene trees and so may not recover the true species tree [47]. While it may be useful also to develop phylogenies based on nuclear markers, there are several reasons why the use of whole mitochondrial genomes provide good indicators of species history. The mitochondrial genome, being haploid and lacking recombination, effectively has four-fold lower coalescence times relative to diploid nuclear genes. This gives much greater phylogenetic resolution than slower evolving nuclear genes. Hence mitochondrial genomes are expected to be good indicators of species history.

Our phylogeny estimated from the WG dataset (Figure 1) is well resolved except for the relative positions of $R$. leucopus and $R$. praetor. The top six observed tree topologies (Table 4) have one or the other of these two species diverging earliest in the Australo-Papuan clade. 


\begin{tabular}{lc}
\hline Clade & Median tMRCA [95\% HPD] \\
\hline Australo-Papuan & $1.66[1.07-2.46]$ \\
R. fuscipes, R. leucopus, R. lutreolus, R. praetor, R. sordidus, R. tunneyi and R. villosissimus & $1.55[0.96-2.25]$ \\
R. fuscipes, R. leucopus, R. lutreolus, R. sordidus, R. tunneyi and R. villosissimus & $1.35[0.86-1.97]$ \\
R. fuscipes, R. lutreolus, R. sordidus, R. tunneyi and R. villosissimus & $0.95[0.56-1.35]$ \\
R. lutreolus, R. sordidus, R. tunneyi and R. villosissimus & $0.84[0.49-1.20]$ \\
R. lutreolus, R. sordidus, and R. villosissimus & $0.49[0.28-0.73]$ \\
R. sordidus, and R. villosissimus & \\
\hline
\end{tabular}

Median estimates (Mya) with 95\% credible limits for the MRCA of the Australo-Papuan clade of Rattus species and the divergences within that clade (Figure 1).

Both species diverge earlier than any of the Australian native rats and there is marginally more support for $R$. praetor diverging earliest. This phylogeny clarifies the relationships among the rats of the Rattus fuscipes species group and is consistent with the taxonomy of Musser and Carleton [1]. The occurrence of $R$. villosissimus and $R$. sordidus as sister species in a shallow divergence is concordant with the phenetic results of Taylor et al. [13], and the phylogenies of Baverstock [14] and Aplin [17]. The more recent divergence of $R$. lutreolus compared with $R$. tunneyi differs from these earlier phylogenies where $R$. lutreolus is either basal to $R$. tunneyi $[13,17]$ or in an unresolved polytomy that includes $R$. fuscipes and $R$. leucopus [14]. In our phylogeny the position of $R$. lutreolus within the Australian native rat clade supports Aplin's [17] view that this rat is more closely related to Australian than to New Guinean rats. The position of $R$. fuscipes basal in our Australian native rat clade but a more recent divergence than that of $R$. leucopus differs from the results of Taylor et al. [13] whose phenogram shows $R$. fuscipes within a clade of New Guinean rats and diverging earlier than the New Guinean species $R$. leucopus.

Our choice of samples for whole mitochondrial genome analysis was influenced by our confidence in the correct assignment of species to those samples. Where there was congruence between the named species and the phylogenetic clades we were confident in the identifications. In Robins et al. [4] we discussed the difficulties that arose when OTUs contained heterogeneous collections of named species. These names were assigned by either the museum or the collector. The source of such heterogeneity could be misidentification, faulty taxonomy due to the plethora of synonyms used for Rattus species, hybridisation, incomplete lineage sorting or some combination of the above. Pagès et al. [11], although they explicitly avoided rats from the AustraloPapuan clade, reported a similar problem. They identified a heterogeneous clade (R3) that was similar to, and where they incorporated data from Robins et al. [4], essentially the same as the diardii clade (Figure 2) therein. In this paper we again resolved the diardii clade. When Pagès et al. [11] considered the same species as we, their genetic based species identifications are largely congruent with ours. This finding supports the usefulness of shorter mitochondrial sequences for species identification. Of the 14 Rattus specimens we used for the whole mitochondrial genomes, 11 were from homogeneous clades. The exceptions were the exulans clade which from a total of 21 samples contained two almost certainly misidentified samples and two New Guinean clades PNG I and verecundus. Our New Guinean species clades are all problematic and to resolve the conflict DNA is needed from specimens that have accurate morphological identifications. All six of the specimens of $R$. praetor that we have processed fell into the PNG I clade and we used one of these samples to represent the clade. At this time we have insufficient information to account for the heterogeneity of this clade although some possibilities are discussed in Robins et al. [4]. The Australian Rattus specimens on the other hand all fell into well supported homogeneous clades thus the phylogenetic assignment of species was in agreement with that of the museum or collector.

Our cytochrome $b$ analyses generally gave robust species clades. Further, the Australo-Papuan rats were in a well-supported and monophyletic clade in all analyses. However, the relationships within Rattus sensu stricto [24], especially among Bandicota, Berylmys, the Philippine endemics (Tarsomys, Limnomys and Rattus everetti) and the main Asian and Australo-Papuan clades of Rattus, were poorly resolved. On the other hand, the deeper relationships within Rattus sensu lato were wellsupported. Pagès et al. [11] stated that they did not obtain a robust phylogeny when they analysed cytochrome $b$ alone. Interestingly, even when they analysed three concatenated genes (one nuclear and two mitochondrial) they were unable to place Bandicota conclusively within or sister to Rattus. Thus the currently available data is insufficient to address the question of monophyly within Rattus as a whole.

The ancestral node in the Australo-Papuan clade differed with the genomic regions used. The inclusion of the hypervariable D-loop (Figures 2B and 3) moved the 
root deeper into the clade, relative to its placement when the whole genome was used (Figure 1) or when the MRCA of Rattus was constrained (Figure 2A). It is likely that the differences arise from substitution rate differences between D-loop and the rest of the genome. The hypervariable nature of the D-loop, with the potential for substitution saturation, also contributed to the inflation (two- to three-fold increase) of dates of divergence in the unconstrained BEAST tree based on the 3G dataset compared with that of the WG dataset.

Although the deeper branching orders are uncertain within the Australo-Papuan clade for the $3 \mathrm{G}$ and the 3G-WG phylogenies (Figures 2 and 3) the Australian native Rattus species, including the newly added $R$. lutreolus and $R$. villosissimus samples fall into well differentiated clades reinforcing the usefulness of shorter mitochondrial DNA sequences for species identification [48]. The position of two samples from New Guinea, misidentified as $R$. rattus and labelled as OTU rattus II in Figures 2A and 2B, as sister to $R$. villosissimus which is in turn sister to $R$. sordidus suggests that these samples are in fact $R$. villosissimus. The usefulness of these shorter sequences, however, to elucidate deeper evolutionary relationships or to date divergences is expected to be limited given the difficulties in inferring species relationships for rapid radiations $[37,38]$. The deeper branches of the Australo-Papuan clade in Figures 2 and 3 are all quite short, indicative of a period of rapid diversification, but the branching pattern varies with the methods used and no one pattern is well supported. It is possible that the inclusion of one or more nuclear markers could help resolve this pattern and is an approach to be pursued in the future, however, given their greater effective population size and longer coalescence times, nuclear genes are likely to be less powerful in resolving the order of divergences. They will, however, provide evidence regarding whether mitochondrial gene trees provide accurate estimates of the deeper species relationships [e.g., [49]].

The dates inferred from the WG dataset analysis are congruent with earlier studies. Jansa et al. [9], using just the IRBP nuclear gene, estimated a date of 3-4 Mya for the origin of a clade containing $R$. exulans, $R$. tanezumi and, $R$. preator (sic). This is the equivalent of our 2.7 Mya split between the Asian and the Australo-Papuan clade (See Figure 1). When Jansa et al. [9] included both IRBP and cyt $b$ data in their analysis the age of the nodes increased three fold and when cyt $b$ alone was used the ages increased four fold. We had a similar problem with the $3 \mathrm{G}$ dataset which when analysed in BEAST, without the constraints described in the methods, returned dates that were almost three fold older than those returned by the WG dataset. It is worth noting also that we observed slightly older dates $\sim 4.6$ Mya (unpublished data) for the Asian/Australo-Papuan split on standard (ACGT) coding, compared with 23.51 Mya obtained when we RY coded our 2008 [5] whole mitochondrial dataset. Rowe et al. [10] included three Rattus sp. in their analyses of the old endemic murines of the Sahul. They recovered evidence for the Asian/AustraloPapuan split with $R$. leucopus and $R$. villosissimus forming a sister pair relative to $R$. norvegicus. Although they did not report a chronogram, we can obtain a rough estimate of the age of the MRCA for Rattus from their Figure 4. The depth of the MRCA of Rattus was $26 \%$ of the depth of the closest dated node (B) in its ancestry (their Table 1), giving an estimated age of 2.52 (2.26 2.81) Mya. This date is concordant with our estimate of 2.7 Mya.

Our dates are also consistent with the earlier study of Robins et al. [5]. Whilst the node dates obtained from our WG BEAST analysis are all slightly younger than the equivalent ones from the previous study they are within the $95 \%$ credible intervals of the 2008 study. Since we have increased the sample size of the Australo-Papuan clade representation from one to eight the dates we report here are more reliable. These younger dates align more closely with those from the L1 (LINE1 , long interspersed repeated) retrotransposable elements study of Verneau et al. [24] who estimated the timing of several major speciation events within Rattus sensu stricto of which the two most recent are relevant to our study. The first at $\sim 2.7$ Mya gave rise to five lineages one of which led to $R$. fuscipes (the only representative of the Australian and New Guinean rats in their study) and another led to Asian Rattus species. This divergence is the equivalent of the split between our Asian and Australo-Papuan clades also dated at $\sim 2.7$ Mya.

The five lineage speciation events described by Verneau et al. [24] and the origin of the Australo-Papuan and Asian clades all estimated at $\sim 2.7$ Mya coincided with Pleistocene Ice Age events. The onset of the Pleistocene glaciation cycles was at about 3 Mya [50] and by 2.6 Mya the Pleistocene Ice Age was well established [51]. Major changes occurred in climate and available land in the region encompassing Island Southeast Asia, New Guinea and Australia. Such changes, for example at the highest glacial maxima New Guinea, Australia, and the islands of Misool, Waigeo, the Aru archipelago and Tasmania formed a single land mass[18], would have provided routes for dispersal and subsequently opportunities for speciation in Rattus. Also during the same time period, there was considerable dispersal of marsupials (bandicoots, dasyurids, phalangerid possums, and kangaroos) between New Guinea and Australia but diversification was more limited [52]. We can speculate that there was greater niche overlap between the newly 
migrated and endemic marsupials while Rattus was not so ecologically constrained. Glacial cycling with some 20 cycles in the last two million years continued to contribute to ongoing climate and sea level changes $[17,50]$ and during this time radiation also continued in Rattus.

The more recent speciation events described by Verneau et al. [24] were a radiation in the Asian Rattus clade. Verneau et al's [24] study included 12 Asian Rattus species and four of these are represented in our study; $R$. norvegicus, $R$. exulans $R$. rattus and $R$. tane$z u m i$. Their divergence estimates were in the time period 1.2 Mya to about 0.5 Mya. Our results are reasonably concordant with but probably more reliable than theirs because their analysis assumed a fixed rate molecular clock. In their L1 study the timing of the divergence of the $R$. norvegicus lineage at $\sim 1.8 \mathrm{Mya}$ compares with $\sim 2.3$ Mya in our study; $R$. exulans at $\sim 1$ Mya compares with our $\sim 1.7$ Mya and the divergence between $R$. rattus and $R$. tanezumi at 0.5 Mya compares with ours at $\sim 0.3$ Mya. The divergences in our Australo-Papuan clade occur over a similar time period (1.7 Mya - $0.5 \mathrm{Mya})$ as that in the Asian clade. $R$. praetor diverged at $\sim 1.7$ Mya, $R$. leucopus at $\sim 1.6$ Mya, $R$. fuscipes at $\sim 1.4$ Mya, $R$. tunneyi at $\sim 1$ Mya, $R$. lutreolus at $\sim 0.9$ Mya and the sister species $R$. sordidus and $R$. villosissimus at $\sim 0.5$ Mya. Despite the uncertainty in assigning the order of the deepest divergences in our Australo-Papuan clade, which is exacerbated by the relatively short time frame of $\sim 0.2 \mathrm{My}$ in which they occurred, the overall pattern from the WG and the 3G datasets suggests that the founding Rattus lineages reached New Guinea by at least 1.7 Mya and Australia by 1.4 Mya with further diversification occurring in both areas between $\sim 1-0.5$ Mya.

When sea levels were as little as $10 \mathrm{~m}$ below present land bridges would have connected New Guinea and Australia at the Torres Strait [53]. These land bridges which have occurred intermittently have also provided opportunities in more recent times for the reinvasion and subsequent isolation of rats between New Guinea and Australia in the Torres Strait area. In our phylogeny $R$. sordidus and $R$. villosissimus diverged most recently in the Australian Rattus clade. This position is consistent with an Australian origin of $R$. sordidus. In contrast the $R$. leucopus lineage diverged earlier than any of the native Australian rats thus supporting a New Guinean origin. The recent split between the Australian and New Guinean sister lineages at $\sim 0.6$ Mya suggests an invasion of Australia by $R$. leucopus followed by isolation. Our findings, therefore, support the Torres Strait faunal interchange hypothesis first put forward by Tate $[15,16]$ with later support from Taylor and Horner [12], Taylor et al. [13] and Aplin [17].

\section{Conclusions}

Although Rattus is a problematic genus and further investigations of the Australo-Papuan group are needed to resolve the relationships of the Rattus species of New Guinea we have clarified the pattern and timing of divergences among the Australian rats. Our whole mitochondrial genome analyses are concordant with Musser and Carleton's [1]Rattus fuscipes species group and resolve the positions of $R$. fuscipes and $R$. lutreolus within it. Our findings suggest that Rattus invaded Australia from New Guinea about 1.4 Mya and that this was followed by a period of rapid speciation. Our results also support and suggest a date of $\sim 0.5$ Mya for the hypothesized $[15,16]$ more recent invasions across Torres Strait of R. sordidus into New Guinea and R. leucopus into Australia.

\section{Additional material}

Additional file 1: WG_partitioned.nxs. The whole mitochondrial genome alignment partitioned into the three codon positions, and RNA stem and loop regions.

Additional file 2: 3G_partitionedByRegion_RY_encoded.nxs. The 3 gene regions (Cyt $\mathrm{B}, \mathrm{COI}$ and $\mathrm{D}$-loop) alignment partitioned into codon positions 1 and 2, codon position 3 as RY and all other positions.

Additional file 3: 3G_partitioned.nxs. The 3 gene regions alignment with each gene as a separate but unencoded partition.

Additional file 4: Sequence labels\&accessions.xls. Summary of sample information: sequence labels, species names and GenBank accessions.

Additional file 5: 3G-WG_partitionedByRegion_RY_encoded.nxs. The 3 gene regions alignment extracted from the specimens in the WG dataset.

Additional file 6: Rodent_cytb_full.nex. The full alignment for the cytochrome $b$ gene sequences of Rattus and closely related genera. Each sequence is labelled with its GenBank accession number followed by its species name.

Additional file 7: Rodent_cytb_full_tre.pdf. Bayesian inference phylogram of the full cytochrome $b$ dataset with the terminal nodes collapsed.

Additional file 8: Rodent_cytb_full.tre

\section{Acknowledgements}

We thank Steve Donnellan and the South Australian Museum for tissue samples. We thank David Penny and Peter Lockhart for discussions and support throughout the project and for advice on the manuscript. We thank two anonymous reviewers for helpful comments. This research was funded by the Allan Wilson Centre for Molecular Ecology and Evolution and the Marsden Fund of New Zealand.

\section{Author details}

'Department of Anthropology and School of Biological Sciences, The University of Auckland, Auckland, New Zealand. ${ }^{2}$ Institute of Molecular Biosciences, Massey University, Palmerston North, New Zealand. ${ }^{3}$ Research School of Biology, Australian National University, Canberra, Australia. ${ }^{4}$ Allan Wilson Centre for Molecular Ecology and Evolution and Institute of Molecular Biosciences Massey University, Palmerston North, New Zealand. ${ }^{5}$ Allan Wilson Centre for Molecular Ecology and Evolution and Department of Anatomy and Structural Biology, Otago School of Medical Sciences, Dunedin, New Zealand. ${ }^{6}$ Allan Wilson Centre for Molecular Ecology and 
Evolution, Bioinformatics Institute and School of Biological Sciences, The University of Auckland, Auckland, New Zealand.

\section{Authors' contributions}

JR and PM designed the project, planned and conducted lab work. All authors analysed data and contributed to the writing and editing of the manuscript. All authors approved the final manuscript.

Received: 15 July 2010 Accepted: 2 December 2010 Published: 2 December 2010

\section{References}

1. Musser GG, Carleton MD: Family Muridae. In Mammal Species of the World: A Taxonomic and Geographic Reference. Volume 2.. 3 edition. Edited by: Wilson DE, Reeder DM. Baltimore: The John Hopkins University Press; 2005:894-1531

2. Watts C, Baverstock PR: Evolution in the Murinae (Rodentia) assessed by microcomplement fixation of albumin. Aust J Zool 1995, 43:105-118.

3. Hall R: The plate tectonics of Cenozoic SE Asia and the distribution of land and sea. In Biogeography and Geological Evolution of SE Asia. Edited by: Hall R, Holloway JD. Leiden: Backhuys Publishers; 1998:99-131.

4. Robins JH, Hingston M, Matisoo-Smith E, Ross HA: Identifying Rattus species using mitochondrial DNA. Mol Ecol Notes 2007, 7:717-729.

5. Robins JH, McLenachan PA, Philips MJ, Craig L, Ross HA, Matisoo-Smith E: Dating of divergences within the Rattus genus phylogeny using whole mitochondrial genomes. Mol Phylogenet Evol 2008, 49:460-466.

6. Innes JG: Ship rat. In The Handbook of New Zealand Mammals. Edited by: King CM. Auckland, Melbourne, Oxford, New York: Oxford University Press; 1990:206-225.

7. Matisoo-Smith E, Roberts RM, Irwin GJ, Allen JS, Penny D, Lambert DM: Patterns of prehistoric human mobility in Polynesia indicated by mtDNA from the Pacific rat. Proc Natl Acad Sci USA 1998, 95:15145-15150.

8. White PJ, Clark G, Bedford S: Distribution, present and past, of Rattus praetor in the Pacific and its implications. Pacific Science 2000 54(2):105-117.

9. Jansa SA, Barker FK, Heaney LR: The pattern and timing of diversification of Philippine endemic rodents: evidence from mitochondrial and nuclear gene sequences. Syst Biol 2006, 55(1):73-88.

10. Rowe KC, Reno ML, Richmond DM, Adkins RM, Steppan SJ: Pliocene colonization and adaptive radiations in Australia and New Guinea (Sahul): Multilocus systematics of the old endemic rodents (Muroidea: Murinae). Mol Phylogenet Evol 2008, 47:84-101.

11. Pagès $M$, Chaval $Y$, Herbreteau V, Waengsothorn $S$, Cosson J-Fo, Hugot JP, Morand S, Michaux J: Revisiting the taxonomy of the Rattini tribe: a phylogeny-based delimitation of species boundaries. BMC Evolutionary Biology 2010, 10:184

12. Taylor JM, Horner E: Results of the Archbold expeditions. No 98. Systematics of native Australian Rattus (Rodentia, Muridae). Bull Am Mus Nat Hist 1973, 150:1-130

13. Taylor JM, Calaby JH, Smith SC: Native Rattus, land bridges, and the Australian region. J Mammal 1983, 64(3):463-475.

14. Baverstock PR, Adams M, Watts CHS: Biochemical differentiation among karyotypic forms of Australian Rattus. Genetica 1986, 71:11-22.

15. Tate GHH: Results of the Archbold expeditions. No. 65. Bull Am Mus Nat Hist 1951, 97:183-430.

16. Tate GHH: Results of the Archbold expeditions. No. 66. Mammals of Cape York Peninsula, with notes on the occurrence of rain forest in Queensland. Bull Am Mus Nat Hist 1952, 98:563-616.

17. Aplin KP: Ten million years of rodent evolution in Australasia: Phylogenetic evidence and a speculative historical biogeography. In Evolution and Biogeography of Australasian Vertebrates. Edited by: Merrick JR, Archer M, Hickey GM, Lee MSY. Oatlands: Auscipub Pty Ltd; 2006:707-744.

18. van Oosterzee P: Drawing the Wallace Line. In Evolution and Biogeography of Australasian Vertebrates. Edited by: Merrick JR, Archer M, Hickey GM, Lee MSY. Oatlands: Auscipub Pty Ltd; 2006:95-110.

19. Schlick NE, Jensen-Seaman Ml, Orlebeke K, Kwitek AE, Jacob HJ, Lazar J: Sequence analysis of the complete mitochondrial DNA in 10 commonly used inbred rat strains. Am J Physiol Cell Physiol 2006, 291(6):C1183-C1192.

20. McComish BJ, Hills SFK, Biggs PJ, Penny D: Index-free de novo assembly and deconvolution of mixed mitochondrial genomes. Genome Biol Evol 2010, 2:410-424.
21. Zerbino DR, Birney E: Velvet: Algorithms for de novo short read assembly using de Bruijn graphs. Genome Research 2008, 18:821-829.

22. Drummond AJ, Ashton B, Cheung M, Heled J, Kearse M, Moir R, StonesHavas S, Thierer T, Wilson A: Geneious. 2009 [http://www.geneious.com/, v4.8.

23. Phillips MJ, Gibb GC, Crimp EA, Penny D: Tinamous and Moa flock together: mitochondrial genome sequence analysis reveals independent losses of flight among Ratites. Syst Biol 2010, 59(1):90-107.

24. Verneau O, Catzeflis F, Furano AV: Determining and dating recent rodent speciation events by using L1 (LINE-1) retrotransposons. Proc Natl Acad Sci USA 1998, 95:11284-11289.

25. Swofford DL: PAUP*: Phylogenetic analysis using parsimony ${ }^{*}$ and other methods). Sunderland, MA, USA: Sinauer " 42002.

26. Stamatakis A: RAxML-VI-HPC: Maximum-likelihood-based phylogenetic analyses with thousands of taxa and mixed models. Bioinformatics 2006 , 22:2688-2690.

27. Guindon S, Gascuel O: A simple, fast, and accurate algorithm to estimate large phylogenies by maximum likelihood. Syst Biol 2003, 52(5):696-704

28. Huelsenbeck JP, Ronquist F: MRBAYES: Bayesian inference of phylogeny. Bioinformatics 2001, 17:754-755.

29. Rambaut A, Drummond AJ: Tracer. 2007 [http://beast.bio.ed.ac.uk/Tracer], v1.5.

30. Kishino $\mathrm{H}$, Hasegawa $\mathrm{M}$ : Evaluation of the maximum likelihood estimate of the evolutionary tree topologies from DNA sequence data, and the branching order in Hominoidea. J Mol Evol 1989, 29:170-179.

31. Shimodaira $\mathrm{H}$ : An approximately unbiased test of phylogenetic tree selection. Syst Biol 2002, 51(3):492-508.

32. Shimodaira H, Hasegawa M: CONSEL: for assessing the confidence of phylogenetic tree selection. Bioinformatics 2001, 17(12):1246-1247.

33. Drummond AJ, Rambaut A: BEAST: Bayesian evolutionary analysis by sampling trees. BMC Evolutionary Biology 2007, 7:214

34. Drummond AJ, Ho SYW, Phillips MJ, Rambaut A: Relaxed phylogenetics and dating with confidence. PLoS Biology 2006, 4(5):e88

35. Benton MJ, Donoghue PCJ: Paleontological evidence to date the tree of life. Mol Biol Evol 2007, 24(1):26-53.

36. Steppan SJ, Adkins RM, Anderson J: Phylogeny and divergence-date estimates of rapid radiations in muroid rodents based on multiple nuclear genes. Syst Biol 2004, 53(4):533-553.

37. Degnan JH, Rosenberg NA: Discordance of species trees with their most likely gene trees. PLOS Genetics 2006, 2(5):762-768.

38. Shavit L, Penny D, Hendy MD, Holland BR: The problem of rooting rapid radiations. Mol Biol Evol 2007, 24(11):2400-2411.

39. Arnason U, Gullberg A, Janke A: Mitogenomic analyses provide new insights into cetacean origin and evolution. Gene 2004, 333:27-34.

40. Cao Y, Fujiwara M, Nikaido M, Okada N, Hasegawa M: Interordinal relationships and timescale of eutherian evolution as inferred from mitochondrial genome data. Gene 2000, 259:149-158.

41. Gibson A, Gowri-Shankar V, Higgs PG, Rattray M: A Comprehensive analysis of mammalian mitochondrial genome base composition and improved phylogenetic methods. Mol Biol Evol 2005, 22(2):251-264.

42. Phillips MJ, McLenachan PA, Down C, Gibb GC, Penny D: Combined mitochondrial and nuclear DNA sequences resolve the interrelations of the major Australasian marsupial radiations. Syst Biol 2006, 55(1):122-137.

43. Phillips MJ, Penny D: The root of the mammalian tree inferred from whole mitochondrial genomes. Mol Phylogenet Evol 2003, 28:171-185

44. Harrison GL, McLenachan PA, Phillips MJ, Slack KE, Cooper A, Penny D: Four new avian mitochondrial genomes help get to basic evolutionary questions in the late Cretaceous. Mol Biol Evol 2004, 21(6):974-983.

45. Arnason U, Gullberg A, Janke A, Joss J, Elmerot C: Mitogenomic analyses of deep gnathostome divergences: a fish is a fish. Gene 2004, 333:61-70

46. Bleidorn C, Eeckhaut I, Podsiadlowski L, Schult N, McHugh D, Halanych KM, Milinkovitch MC, Tiedemann R: Mitochondrial genome and nuclear sequence data support Myzostomida as part of the annelid radiation. Mol Biol Evol 2007, 24(8):1690-1701.

47. Maddison WP: Gene trees in species trees. Syst Biol 1997, 46(3):523-536.

48. Ross HA, Murugan S, Li WLS: Testing the reliability of genetic methods of species identification via simulation. Syst Biol 2008, 57(2):216-230.

49. Spinks $P Q$, Shaffer $H B$ : Conflicting mitochondrial and nuclear phylogenies for the widely disjunct Emys (Testudines: Emydidae) species complex and what they tell us about biogeography and hybridization. Syst Biol 2009, 58:1-20. 
50. Lambeck K, Esat TM, Potter EK: Links between climate and sea levels for the past three million years. Nature 2002, 419:199-206.

51. White ME: Environments of the geological past. In Evolution and Biogeography of Australasian Vertebrates. Edited by: Merrick JR, Archer M, Hickey GM, Lee MSY. Oatlands: Auscipub Pty Ltd; 2006:17-50.

52. Kirsch JAW, Lapointe FJ, Springer MS: DNA-hybridisation studies of marsupials and their implications for metatherian classification. Aust $J$ Zool 1997, 45:211-280.

53. Voris HK: Maps of Pleistocene sea levels in Southeast Asia: shorelines, river systems and time durations. J Biogeogr 2000, 27:1153-1167.

doi:10.1186/1471-2148-10-375

Cite this article as: Robins et al:: Evolutionary relationships and

divergence times among the native rats of Australia. BMC Evolutionary

Biology 2010 10:375.

Submit your next manuscript to BioMed Central and take full advantage of:

- Convenient online submission

- Thorough peer review

- No space constraints or color figure charges

- Immediate publication on acceptance

- Inclusion in PubMed, CAS, Scopus and Google Scholar

- Research which is freely available for redistribution

Submit your manuscript at www.biomedcentral.com/submit
Ciomed Central 